\title{
Women gaze behaviour in assessing female bodies - the effects of clothing, body size, own body composition and body satisfaction
}

Amelia Cundall, Kun Guo

School of Psychology, University of Lincoln, UK

\begin{abstract}
Often with minimally clothed figures depicting extreme body sizes, previous studies have shown women tend to gaze at evolutionary determinants of attractiveness when viewing female bodies, possibly for self-evaluation purposes, and their gaze distribution is modulated by own body dissatisfaction level. To explore to what extent women's body-viewing gaze behaviour is affected by clothing type, dress size, subjective measurements of regional body satisfaction and objective measurements of own body composition (e.g., chest size, body mass index, waist-to-hip ratio), in this self-paced body attractiveness and body size judgement experiment, we compared healthy, young women's gaze distributions when viewing female bodies in tight and loose clothing of different dress sizes. In contrast to tight clothing, loose clothing biased gaze away from the waist-hip to the leg region, and subsequently led to enhanced body attractiveness ratings and body size underestimation for larger female bodies, indicating the important role of clothing in mediating women's body perception. When viewing preferred female bodies, women's higher satisfaction of a specific body region was associated with an increased gaze towards neighbouring body areas, implying satisfaction might reduce the need for comparison of confident body parts; furthermore undesirable body composition measurements were correlated with a gaze avoidance process if the construct was less changeable (i.e. chest size) but a gaze comparison process if the region was more changeable (i.e. body mass index, dress size). Clearly, own body satisfaction and body composition measurements had an evident impact on women’s body-viewing gaze allocation, possibly through different cognitive processes.
\end{abstract}




\section{Introduction}

The human body is one of the most common visual stimuli in our social surrounding, and viewing other people often involves conscious or unconscious judgement of their body attractiveness (Pawlowski \& Dunbar, 1999). The subsequent question of what drives female body attractiveness judgement or female body perception from women's perspective attracts research interest across a range of disciplines and has wide applications in social (e.g., social behaviour), forensic (e.g., sexual preference) and clinical (e.g., eating disorder) psychology.

From an evolutionary perspective, female attractiveness is centred on reproductive capability, thus its determinants should be indicative of this function. Indeed, factors such as body mass index (BMI), body fat and waist-to-hip ratio (WHR) have been shown to correlate with attractiveness judgements (Singh, 1993; Tovée, Reinhardt, Emery, \& Cornelissen, 1998; Weeden \& Sabini, 2005), and are predictive of both health and fertility (Singh \& Singh, 2011). For instance, slender figures with a low WHR and large breasts are often rated as more attractive and considered for relationships (Singh \& Young, 1995), furthermore these features have been shown to correlate with high fecundity as measured by levels of sex hormones (Jasieńska, Ziomkiewicz, Ellison, Lipson, \& Thune, 2004).

Considering that waist-hip and chest regions transmit diagnostic cues for female body attractiveness judgement, these body features are more likely to attract visual inspection in body-viewing. Indeed, recent eye-tracking studies have observed that in the tasks of free-viewing, body attractiveness and body fat judgement, both male and female viewers demonstrated similar gaze distribution with more gaze allocated at the waist-hip and chest areas (Cornelissen, Hancock, Kiviniemi, George, \& Tovée, 2009; Hall, Hogue, \& Guo, 2011). These observations of both genders using the same visual 
features to assess female body could be accounted for by mate selection theory, which postulates women judge their own attractiveness relative to other women in order to assess their own likelihood for successful mate selection (Buss, 2003) or monitor potential competitors as attractive women have high 'market value' (Hughes, Harrison, \& Gallup, 2004; Pawlowski \& Dunbar, 1999) and pose a greater threat to partner sexual fidelity (O’Connor \& Feinberg, 2012). Indeed, when using image manipulation to create the ideal partner, women demonstrated an accurate idea of what heterosexual men find attractive (Crossley, Cornelissen, \& Tovée, 2012).

This possibility for women to judge their own attractiveness value and monitor competitors might be what drives their gaze patterns when viewing female body images, and suggests a preoccupation with the need for social comparison to establish one's own 'market value' or social learning to acquire tips on how to improve one's 'market value' (Hahn \& Perrett, 2014). This view has been further supported by women's preference for viewing other female bodies. For instance, when presented with erotic and non-erotic images of heterosexual couples, men looked at female bodies significantly longer than male bodies in the picture, whereas women tended to distribute their attention evenly between female and male bodies (Lykins, Meana, \& Strauss, 2008). Similarly, when inspecting sexually explicit photos, the amount of viewing time directed at female bodies was indistinguishable between male and female viewers (Rupp \& Wallen, 2007).

According to social comparison theory which centres on the notion that people have a central desire to evaluate themselves for an accurate representation (Festinger, 1954), the comparison could be either driven by self-improvement and made with those with better abilities (upward comparison) or driven by self-enhancement and made with weaker individuals (downward comparison). When women evaluate female bodies, upward comparisons often occur (possibly due to media influences and societal 
pressures, such as the ideal body with large breasts and a small waist) and could lead to body dissatisfaction or even clinical symptoms such as disordered eating. Equally, dissatisfaction with one's body could increase self-activation and self-concern about meeting standards, and consequently increase proneness to undertake social comparisons (Fiske, 2011; Stice \& Shaw, 2002). Therefore, it is plausible that women's own body satisfaction or dissatisfaction level may bias their assessment of female body and associated gaze distribution. However, previous studies in this area have revealed inconsistent findings (e.g., Blechert, Nickert, Caffier, \& Tuschen-Caffier, 2009; Cho \& Lee, 2013; Glauert, Rhodes, Fink, \& Grammer, 2010; Jiang \& Vartanian, 2012).

On the one hand, many studies have suggested that women with high body dissatisfaction attend to idealised bodies and undertake upward social comparison. For instance, in comparison to women with low body dissatisfaction, women with high body dissatisfaction viewed thin bodies longer than average and overweight bodies (Cho \& Lee, 2013). Patients with Bulimia Nervosa preferred to view slimmer bodies, whereas healthy controls showed similar viewing preference for bodies with high and low BMIs (Blechert et al., 2009). Furthermore, women scoring high on eating disorder symptomology tended to focus on the self-identified "beautiful" body parts on other women, whereas healthy controls focused on the "ugly" body parts (Jansen, Nederkoorn, \& Mulkens, 2005).

However, somewhat contradictory findings by Glauert et al. (2010) revealed that although all women in their study showed an attentional bias towards thin bodies, those with high body dissatisfaction had a reduced bias. Additionally restrained eaters directed the same amount of attention at both thin and overweight bodies, and crucially these viewing patterns did not differ from those of unrestrained eaters (Jiang \& 
Vartanian, 2012). Clearly the effect of body dissatisfaction on women's body-viewing gaze behaviour is not conclusive and requires further exploration. As the majority of these studies focused on women with eating disorders, there is need for more research on non-clinical populations in order to identify any cognitive process which may lead to symptoms of body dissatisfaction, so interventions can be implemented to alleviate them before they manifest further.

Furthermore, the vast majority of research on this topic has only explored global body dissatisfaction, it is unclear how body region dissatisfaction influences gaze to female body regions. This is relevant as body dissatisfaction is unlikely to involve the whole body, what seems more probable is that certain regions of the body drive the feelings of body dissatisfaction. To our knowledge, only one recent publication has examined this research question explicitly. Lykins, Ferris and Graham (2014) found that higher satisfaction with both mid and lower torso regions could predict more gaze at these regions on both idealised and plus sized models, whereas higher dissatisfaction predicted less attention. This apparent avoidance viewing strategy for both the idealised and plus sized models implies the preservation of dissatisfaction feelings, as the opportunity to undertake downward comparisons with the plus sized models was not utilised by the participants. Similar avoidance viewing behaviour has also been noticed in global body dissatisfaction and sexuality research. Specifically, women with high body dissatisfaction or scoring high on sexual inhibition and low on sexual compulsivity preferentially attended to the face and legs rather than chest and waist-hip, areas key to attractiveness and thus likely to be prone to feelings of inadequacy (Hall, Hogue, \& Guo, 2014; Jannelle, Hausenblas, Ellis, Coombes, \& Duley, 2009). It is plausible that this avoidance gaze distribution, possibly correlated with regional body dissatisfaction, is a general viewing behaviour when women inspect female bodies. This possibility was systematically examined in this study. As measurements of BMI, WHR 
and chest size are strong predictors of attractiveness (Singh, 1993; Tovée et al., 1998; Weeden \& Sabini, 2005), when assessing regional body dissatisfaction we included both objective measurements and subjective ratings of body parts.

There are two more factors which may affect the generalisation of previous findings on female body perception and associated viewing behaviour. The first one is the clothing effect. The typical stimuli used in previous research included photographic or computerised images of females in swimwear (Roefs et al., 2008; Lykins et al., 2014), underwear (Jansen et al., 2005), Lycra (Blechert et al., 2009; Jannelle et al., 2009) or nude (Glauert et al., 2010; Horndasch et al., 2012), thus revealing the shape of the figure in great detail. What is yet to be considered is the viewing behaviour for images where the body regions are somewhat ambiguous, such as in everyday clothing. Considering that the visibility of body regions can modify gaze distribution in bodyviewing (e.g., women fixated more on nude versus clothed female images, and removal of clothing biased fixations away from the face to the chest and pelvic areas; Nummenmaa, Hietanen, Santtila, \& Hyönä, 2012), the influence of clothing should not be overlooked. If women demonstrate the same viewing behaviour for female bodies in both tight clothing (with unambiguous regional body cues) and loose clothing (with ambiguous regional body cues), then it could be argued their gaze allocation is driven predominantly by top-down cognitive processes (e.g., knowledge about location of body parts containing task-related information) rather than bottom-up local image saliency (e.g., visibility of local body parts).

The second limiting factor is the use of extreme body sizes (either thin or overweight bodies) in the majority of previous studies. Although such stimulus selection will help to differentiate women's behavioural responses in body perception, it may not truly reflect their preference in body size. Clearly, research on body perception using images of women in everyday clothing with a range of dress sizes would have 
higher ecological validity.

In this eye-tracking study, we aimed to systematically address these identified research limitations in female body perception from women's perspective. To mimic real world situations, we presented high-resolution body images from well-controlled models in a continuum of common dress sizes in both tight and loose clothing, and healthy female viewers were asked to rate the perceived body attractiveness and dress size. Their gaze distributions in body-viewing were then correlated with their behavioural responses, their own body composition measurements (BMI, WHR and chest size) and regional body satisfaction ratings. Guided by previous research, we hypothesised that (1) participants would attend to waist-hip and chest regions for assessing body attractiveness and dress size, and show rating preference for smaller dress sizes; (2) clothing would affect participants' ratings and body-viewing gaze allocation, as loose clothing conceals body regions (e.g., waist-hip) crucial for attractiveness assessment; (3) participants' own body composition and regional body dissatisfaction would affect their gaze allocation to the concerned body regions, possibly showing an avoidance viewing behaviour.

\section{Materials and Methods}

\subsection{Participants}

Advertising through the departmental subject pool, thirty-three female psychology undergraduate students, aged between 18 and 24 years old (19.48 \pm 1.28 , Mean \pm SD), volunteered to participate in this study in return for course credit. All participants reported heterosexual orientation, no history of eating disorders, and had normal or corrected-to-normal visual acuity. Prior to the study, the research purpose, experimental tasks and procedure had been explained to the participants, and written informed consent was obtained from each of them. The Ethical Committee in School of 
Psychology, University of Lincoln approved this study, and all procedures complied with the British Psychological Society Code of Ethics and Conduct.

\subsection{Visual stimuli}

High-resolution fully clothed female body images (computer-generated avatars) were obtained from a free online virtual fitting room website (www.trymetail.com). Measurements typical of UK dress sizes (obtained from www.asos.com) were entered into the software to produce full body images depicting seven dress sizes ranging from UK6 to UK18 (size 6, 8, 10, 12, 14, 16 and 18; height measurements were standardised at $165 \mathrm{~cm}$ ). These sizes were chosen based on those commonly found in high street stores. The faces of four Caucasian models were chosen to represent each dress size. Each model was of a similar age, had the same hairstyle and similar facial expression with no distinctive facial or body markings, and was presented twice with different clothing style (one in loose clothing and one in tight clothing). Of the four models, two were viewed at a full body frontal view, and two at a $45^{\circ}$ full body mid-profile view (see Fig.1 for image examples of size 6 and 18). In total, 56 body images (8 images per size $\times 7$ dress sizes) were created for testing. The size (width) of the images were determined by the dress size $\left(200-222 \times 663\right.$ pixels, $\left.7.69^{\circ}-8.54^{\circ} \times 25.5^{\circ}\right)$. 

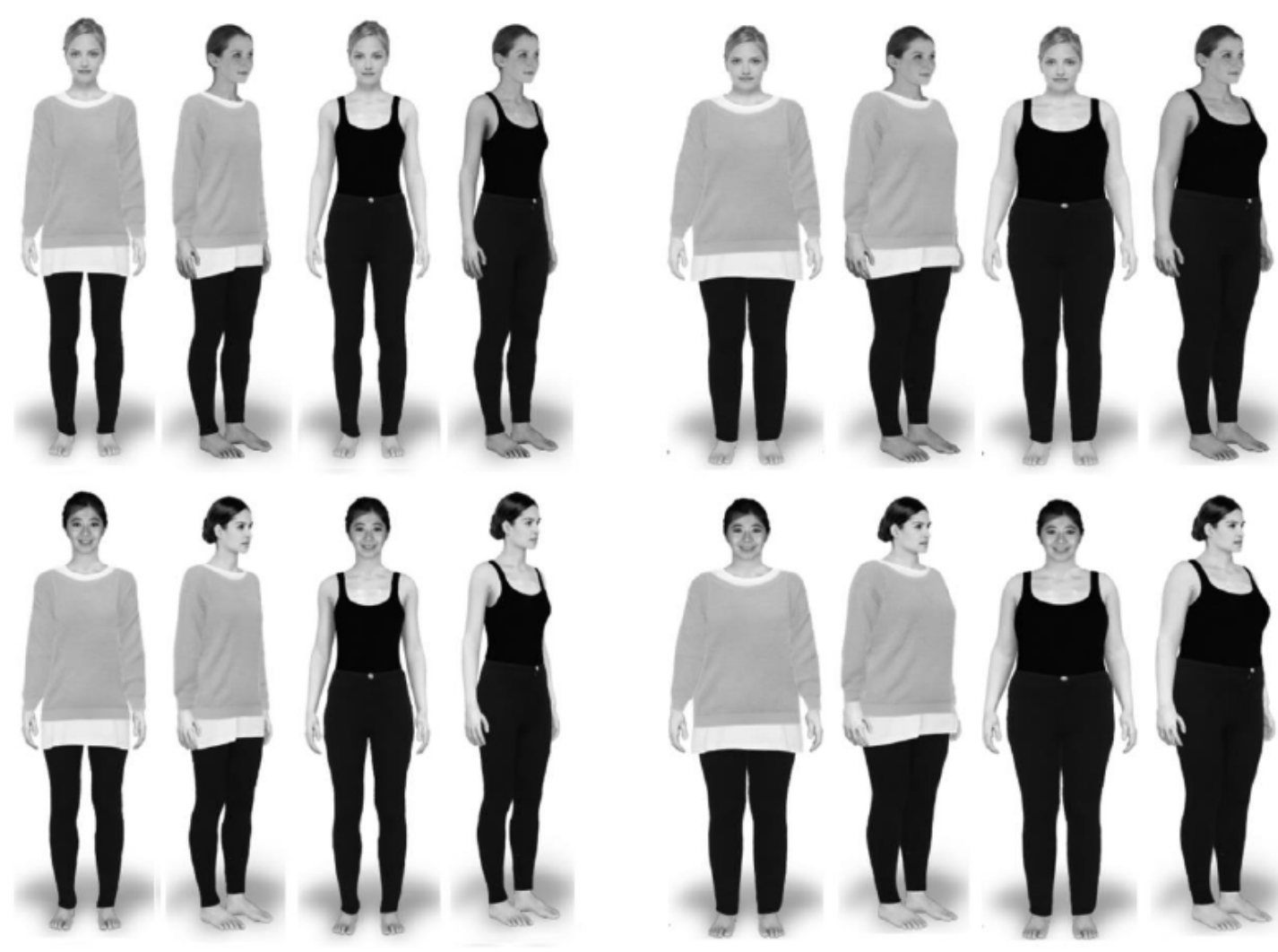

Figure 1. Examples of female body images in UK dress size 6 (left) and 18 (right).

The digitized grey-scale body images were presented through a ViSaGe graphics system (Cambridge Research Systems, UK) and displayed on a noninterlaced gamma-corrected colour monitor (30 cd/m² background luminance, 100 Hz frame rate, Mitsubishi Diamond Pro2070SB) with the resolution of $1024 \times 768$ pixels. At a viewing distance of $57 \mathrm{~cm}$, the monitor subtended a visual angle of $40^{\circ} \times 30^{\circ}$.

\subsection{Procedure}

A self-paced task was used to mimic natural viewing condition. During the eye- tracking experiment the participants sat in a chair with their head restrained by a chin- rest, and viewed the display binocularly. Horizontal and vertical eye positions from the dominant eye (determined through the Hole-in-Card test) were measured using a Video Eyetracker Toolbox with $250 \mathrm{~Hz}$ sampling frequency 
and up to $0.25^{\circ}$ accuracy (Cambridge Research Systems, UK). Eye movement signals were first calibrated by instructing the participant to follow a fixation point (FP, $0.3^{\circ}$ diameter, $15 \mathrm{~cd} / \mathrm{m}^{2}$ luminance) displayed randomly at one of 9 positions $(3 \times 3$ matrix) across the monitor (distance between adjacent FP positions was $\left.10^{\circ}\right)$

After the calibration procedure, the participant pressed the response box to initiate a trial. The trial was started with an FP displayed $10^{\circ}$ left or right to the screen centre to minimize central fixation bias (Tatler, 2007). If the participant maintained fixation for $1 \mathrm{~s}$, the FP disappeared and a testing image was presented at the centre of the screen. During the self-paced presentation, participants were instructed to "rate body attractiveness and body size as accurately and as quickly as possible”, and to respond by pressing a button on the response box (for collecting reaction time data) with the dominant hand followed by a verbal report of the body attractiveness rating on a 9-point scale (1 represents 'not attractive at all' and 9 represents 'extremely attractive'), and body size rating on a scale ranging from UK size 6 to 18. During the testing no feedback was given, and the body images were displayed once in a random order.

Considering that body satisfaction measures might temporally enhance ownbody awareness and consequently affect body-viewing gaze behaviour, the body satisfaction measures were conducted after the eye-tracking task to avoid the potential carryover effects. Participants were required to complete three questionnaires which included (1) Body composition: participants’ weight, height, and waist and hip sizes were measured to calculate body mass index (BMI, weight/height ${ }^{2}$ ) and waist-to-hip ratio (WHR). Participants’ chest cup size and UK dress size were also recorded through self-report measures. (2) Body satisfaction: participants self-rated their satisfaction with each of six body regions (face, breasts, waist, hips, arms and legs) on a 10-point scale, 1 being the most dissatisfied and 10 being the most satisfied. (3) Physical Appearance 
Comparison Scale (PACS; Thompson, Heinberg, \& Tantleff, 1991): PACS is a five item scale used to measure an individual's tendency to use social comparison to evaluate their own appearance. The scale includes items such as "In social situations, I sometimes compare my figure to the figures of other people” and responses range from Never (1) to Always (5). Internal consistency using Cronbach's alpha in the original sample was 0.78 and test-retest reliability was 0.72 (Thompson et al., 1991). Internal consistency for our sample was 0.7 .

\subsection{Data analysis}

All the collected data were analysed off-line. For eye movement data, the software developed in Matlab computed horizontal and vertical eye displacement signals as a function of time to determine eye velocity and position. Fixation locations were then extracted from the raw eye-tracking data using velocity (less than $0.2^{\circ}$ eye displacement at a velocity of less than 20\% (Guo, Mahmoodi, Robertson, \& Young, 2006). To determine gaze allocation within key body regions (Hall, Hogue, \& Guo, 2011), each body was divided into five regions of interest: face (including hair), upper-body (from the base of the neck to the end of the rib cage), waist-hip region (including the stomach, hips, and pubic region), arms (including hands) and legs (including feet). The viewing time allocated to each region was normalised in proportion to the total viewing time sampled in that trial.

A series of repeated measures analysis of variance (ANOVAs) were conducted to examine the effect of dress size and clothing type on participants' body attractiveness and size judgement, and body-viewing gaze allocation. For each ANOVA, Greenhouse-Geisser correction was applied where sphericity was violated, and a Bonferroni adjustment was made for post-hoc multiple comparisons. 


\section{Results}

3.1 Effect of dress size and clothing type on body attractiveness and size judgement

Body attractiveness judgement: to explore to what extent body attractiveness judgements were affected by body size and clothing type, a 7 (dress size) $\times 2$ (clothing) ANOVA was conducted with attractiveness rating score for each dress size as the dependent variable. The analysis revealed significant main effect of dress size $[\mathrm{F}(2.38$, $76)=35.20, \mathrm{p}<0.001, \eta p=0.52$; Fig. 2] with size 18 rated as the least attractive (p $<0.001$ for all comparisons), and larger dress sizes (size 14 and 16) rated less attractive than smaller sizes (size 6, 8, 10 and 12; p $<0.002$ for all comparisons, except no difference between size 6 and 14). Significant effect was also found for clothing [F(1, $32)=10.13, p=0.003, \eta p 2=0.24]$ and interaction between dress size and clothing $[F(6,192)=7.25, p<0.001, \eta p=0.19]$. Specifically, smaller bodies (size 6, 8 and 10) in either loose or tight clothing were rated equally attractive $(\mathrm{t}<0.79, \mathrm{p}>0.43$ for all comparisons, Fig. 2), whereas larger bodies (size 12, 14, 16 and 18) in loose clothing were rated as more attractive than in tight clothing $(\mathrm{t}>2.82, \mathrm{p}<0.008$ for all comparisons).

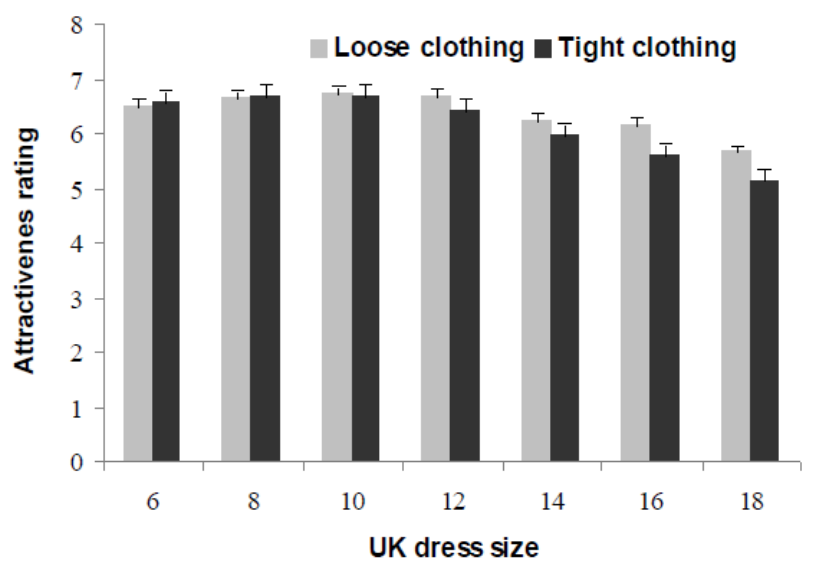


Figure 2. Attractiveness ratings for each dress size and clothing type. Error bars represent standard error of the mean.

Body size judgement: to explore whether body size judgements were affected by dress size and clothing type, a 7 (dress size) $\times 2$ (clothing) ANOVA was conducted with body size rating for each dress size as the dependent variable. The analysis revealed significant main effect of dress size $\left[F(2.15,68.75)=490.95, p<0.001, \eta_{p}^{2}=0.94\right]$ and clothing $\left[F(1,32)=45.97, p<0.001, \eta_{p}{ }^{2}=0.59\right]$, and significant interaction between dress size and clothing $\left[F(6,192)=11.27, p<0.001, \eta_{p}^{2}=0.26\right]$. Specifically, dress sizes 10 to 18 in tight clothing received significantly larger body size ratings than in loose clothing ( $t>3.99, p<0.001$ for all comparisons; Table 1 and Fig. 3). Additionally, smaller dress sizes (size 6 and 8) in both loose and tight clothing were overestimated in body size in comparison to true sizes, whereas larger dress sizes (size 10, 12, 14, 16 and 18) were underestimated $(t>2.13, p<0.04$ for all comparisons, except no difference for size 10 or 12 in tight clothing).

Table 1 Mean body size ratings for each clothing type and dress size (Mean \pm SEM).

\begin{tabular}{ccc} 
Dress Size & $\begin{array}{c}\text { Size rating } \\
\text { (Loose clothing) }\end{array}$ & $\begin{array}{c}\text { Size rating } \\
\text { (Tight clothing) }\end{array}$ \\
\hline $\mathbf{6}$ & $7.65 \pm 0.17$ & $7.82 \pm 0.19$ \\
$\mathbf{8}$ & $8.42 \pm 0.19$ & $8.44 \pm 0.21$ \\
$\mathbf{1 0}$ & $9.27 \pm 0.18$ & $10.02 \pm 0.22$ \\
$\mathbf{1 2}$ & $10.53 \pm 0.21$ & $11.76 \pm 0.25$ \\
$\mathbf{1 4}$ & $11.91 \pm 0.26$ & $12.94 \pm 0.29$ \\
$\mathbf{1 6}$ & $12.68 \pm 0.26$ & $14.3 \pm 0.27$ \\
$\mathbf{1 8}$ & $14.47 \pm 0.29$ & $15.67 \pm 0.29$ \\
\hline
\end{tabular}




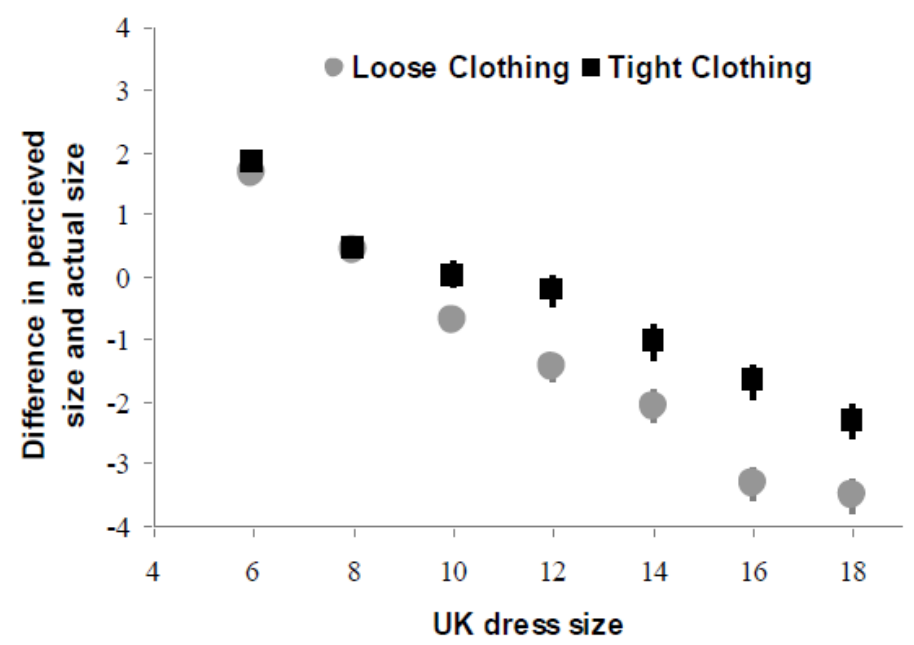

Figure 3. Differences in perceived body size and actual dress size for each clothing type on each dress size. Error bars represent standard error of the mean.

3.1. Effect of dress size and clothing type on body-viewing gaze allocation

To explore whether gaze allocation at individual body regions was affected by dress size and clothing type, 7 (dress size) $\times 2$ (clothing) $\times 5$ (body region) ANOVA was conducted with proportion of viewing time allocated at each body region as the dependent variable. The analysis revealed significant main effect of dress size $\left[F(4.38,140.16)=4.96, p=0.001, \eta_{p}^{2}=0.13\right]$, clothing $\left[F(1,32)=6.23, p=0.02, \quad \eta_{p}^{2}=\right.$ $0.16]$ and body region $\left[F(2.3,73.65)=52.61, p<0.001, \eta_{p}^{2}=0.62\right.$; Fig. 4]. Across all the dress sizes and clothing types, waist-hip region attracted the highest proportion of viewing time (39\% \pm 2 , Mean \pm SEM), followed by head (20\% \pm 3$)$, upper-body $\quad(19 \%$ $\pm 2)$ and legs $(10 \% \pm 1)$. The arms received the lowest proportion of viewing time $\quad(1 \%$ $\pm 0.2)(p<0.02$ for all comparisons).

The analysis also showed significant interaction between dress size $\times$ clothing $\times$ body region $\left[F(10.35,331.11)=2.29, p=0.01, \eta_{p}^{2}=0.07\right.$; Fig. 4]. Specifically, regardless of body size, in comparison with the body regions in loose clothing, the 
waist-hip in tight clothing attracted longer viewing time, whereas the legs attracted shorter viewing time $(t>3.22, p<0.003$ for all comparisons, except for leg region in size 10). For each clothing type, viewing time directed at the waist-hip and leg regions was further modulated by dress size. In loose clothing, the leg region in both size 16 and 18 elicited less viewing time compared to smaller body sizes $(p<0.01$ for all comparisons). In tight clothing, the waist-hip region in size 6 attracted less viewing than size $14(p=0.02)$. No other difference was observed.
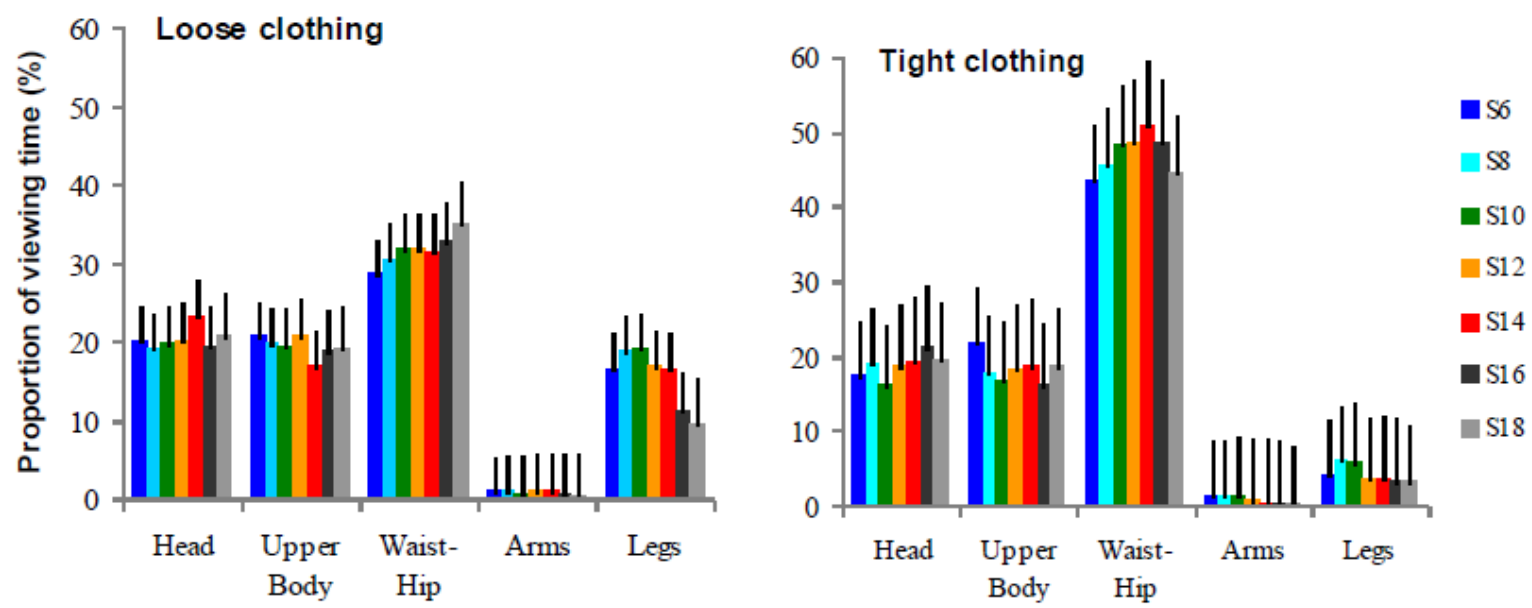

Figure 4. Average proportion of viewing time directed at individual body regions in each dress size with loose clothing (left) and tight clothing (right). Error bars represent standard error of the mean.

3.2. Correlation between body-viewing gaze distribution and body attractiveness and body size judgement?

Despite a negative trend, across clothing types there was no significant correlation between attractiveness scores and rating differences in perceived body size and actual dress size $(r=-.29, p=0.1)$, suggesting attractiveness judgement was not linked with the accuracy of assessing body size. Further Pearson correlation analysis revealed that allocation of viewing time on individual body regions (face, upper-body, waist-hip, arms and legs) did not significantly correlate with body attractiveness or body size ratings ( $p>0.11$ for all comparisons), indicating that as a population, 
women's body-viewing gaze distribution had no direct impact on body attractiveness and body size judgements.

\subsection{Individual differences in body-viewing gaze distribution?}

We then performed a series of correlation analysis to systematically examine to what extent participant's gaze distribution in assessing female bodies (proportion of viewing time at local body regions, such as face, upper-body, waist-hip, arms and legs) were affected by their own body composition (own dress size, chest size, BMI, waisthip ratio) and their satisfaction of own body regions (self-rated regional body satisfaction scores for face, arms, legs, chest, and waist-hip region).

\subsubsection{Objective measurements of own body composition}

Across our participants, their dress sizes ranged from UK6 to UK14 (9.5 \pm 0.4$)$, BMI ranged from 18.50 to 27.68 (21.6 \pm 0.43$)$, chest sizes ranged from cup size A to F with the average of a $C$ cup, and waist-hip ratios ranged from 0.65 to $0.84(0.74 \pm 0.01$, all within the healthy range). To examine to what extent participants' own body composition influenced their viewing behaviour in body perception, Pearson correlations were conducted between these objective body measurements and proportion of viewing time directed at each body region, averaged across all body sizes. When viewing body images in tight clothing, participants' chest size and dress size were positively correlated with the proportion of viewing time directed at the upperbody region ( $r=0.36, p=0.04)$ and leg region ( $r=0.35, p=0.05$; Fig. 5), respectively. No other significant correlations were found. 

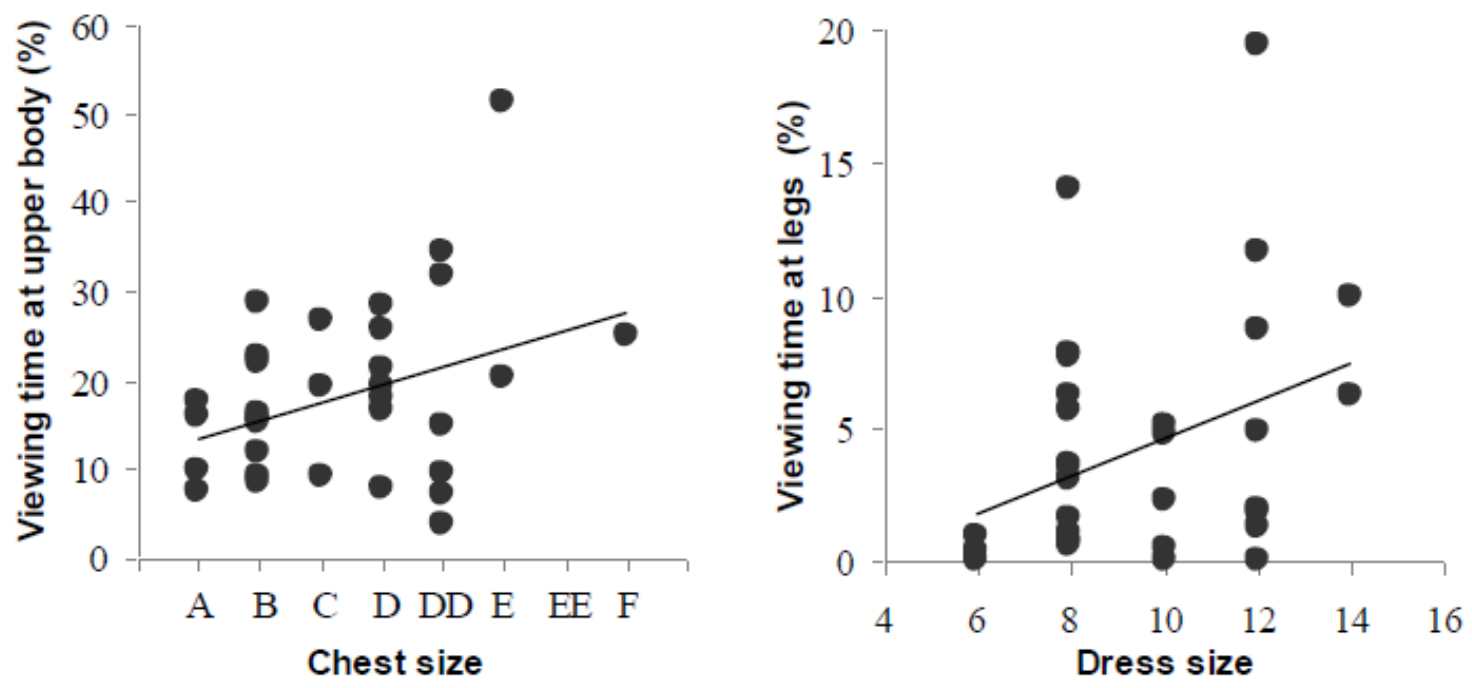

Figure 5. Correlation between participants' chest size and viewing at upper-body region (left), and between dress size and viewing at leg region (right), for tight clothing only.

Although the viewed body size had no clear impact on the correlation analysis between body measurements and viewing behaviour, participants' own preference for body size might play a role. To examine this possibility, correlations were conducted between participants' objective body measurements and proportion of viewing time at each region of their preferred and least preferred body size (determined from their body attractiveness ratings). The analysis showed that participant's chest size and BMI were positively correlated with the viewing time at the upper-body $(r=0.35, p=0.05)$ and legs ( $r=0.34, p=0.05$; Fig. 6) of the preferred body size. No other significant correlations were observed. 

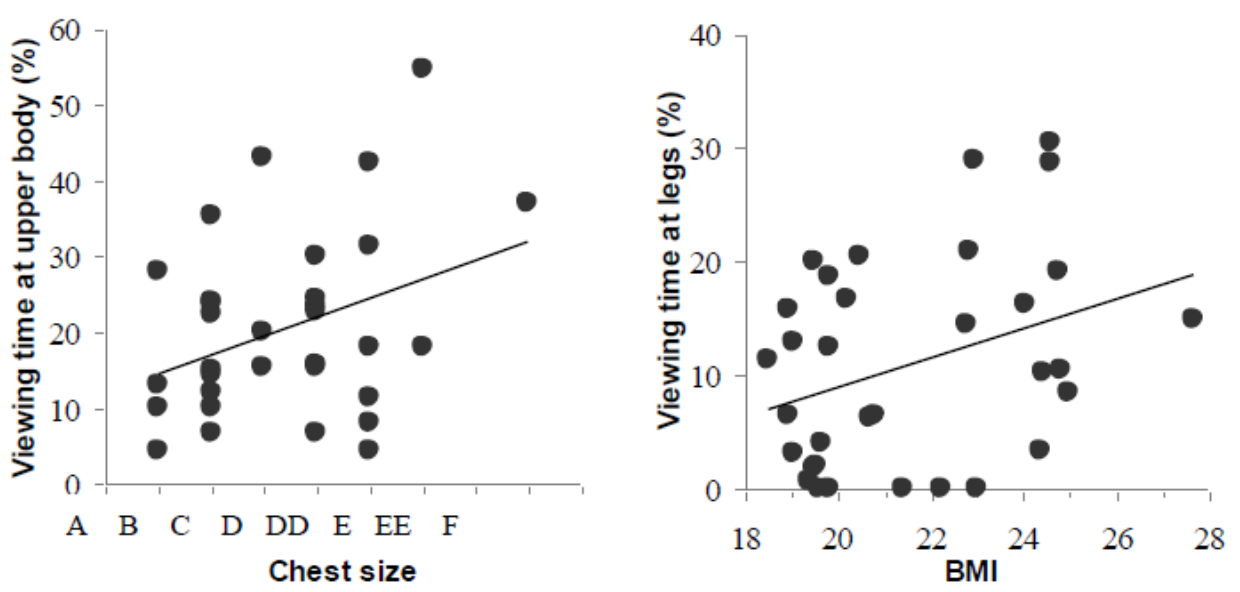

Figure 6. Correlation between participants' chest size and viewing at upper-body region (left), and between BMI and viewing at leg region (right) of the preferred body size.

\subsubsection{Subjective satisfaction of own body composition}

Overall, participants scored similar above-average satisfaction for individual body regions (face $6.85 \pm 0.2$, legs $6.55 \pm 0.28$, arms $6.52 \pm 0.28$, waist-hip $6.5 \pm 0.28$, chest $6.3 \pm 0.3)\left[F(4,128)=0.7, p=0.59, \eta_{p}^{2}=0.02\right]$. Body region ratings were then computed together to produce an overall body satisfaction score of $6.54 \pm 0.18(\alpha=$ 0.62). The participants’ Physical Appearance Comparison Scale (PACS, $16.48 \pm 0.56$ ) was negatively correlated with overall body satisfaction $(r=-0.37, p=0.04)$, indicating those scoring lower in body satisfaction tended to undertake more frequent appearance comparisons. Correlation analysis between these self-rated regional body satisfaction scores and proportions of viewing time directed at each body region for all dress sizes further revealed that the waist-hip satisfaction was positively correlated with the proportion of viewing time allocated at the upper-body region in loose clothing $(\mathrm{r}=$ $0.39, p=0.03)$. No other significant correlations were found.

Correlations were then conducted between participants' self-rated regional body satisfaction scores and the proportion of viewing time at each region of their preferred and least preferred body size. As shown in Fig. 7, when viewing images of the preferred 
body size, arm and leg satisfaction scores were positively correlated with the viewing time at the waist-hip $(\mathrm{r}=0.45, p=0.01)$ and arm region $(\mathrm{r}=0.35, p=0.04)$, respectively. On the other hand, waist-hip satisfaction was positively correlated with the viewing time at the upper-body in both the preferred $(r=0.47, p=0.01)$ and least preferred body size ( $r=0.41, p=0.02$ ). No other significant correlations were observed.
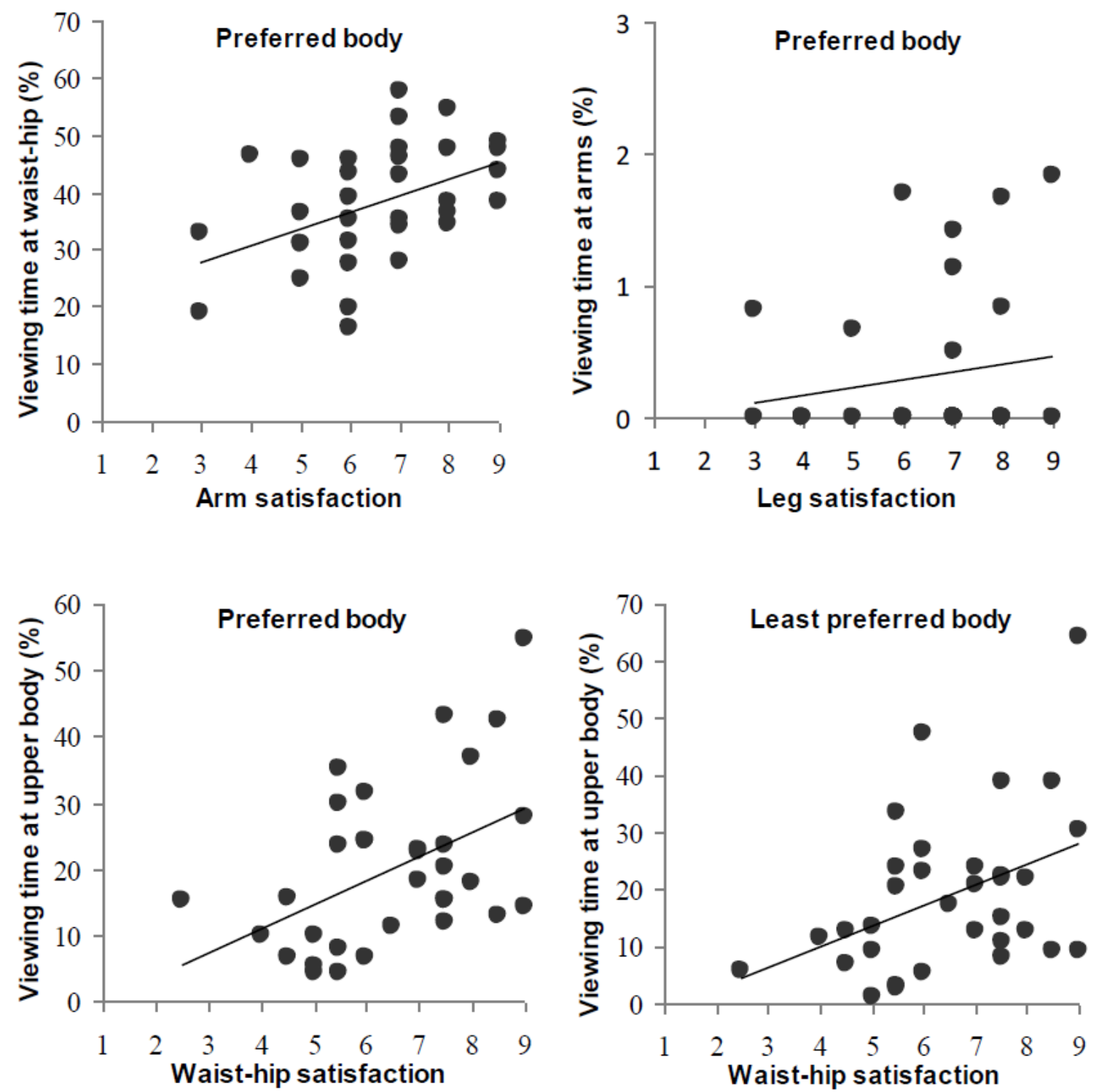

Figure 7. Correlations between subjective body region satisfaction score and proportion of viewing time at body regions in the preferred body size (arm satisfaction and waist- hip viewing, leg satisfaction and arm viewing, waist-hip satisfaction and upper-body viewing) and in the least preferred body size (waist-hip satisfaction and upper-body viewing). 


\section{Discussion}

This exploratory study aimed to advance previous research on female body perception from women's perspective. Specifically, the effect of dress size and clothing type on body attractiveness and body size judgements and associated gaze behaviour were analysed. Additionally the effect of individual differences, including own body satisfaction and body composition, on the body-viewing gaze behaviour was assessed.

4.1. Dress size and clothing type affect body attractiveness and size assessment?

This study revealed that generally larger dress sizes were deemed less attractive. The largest size, UK18, was rated as the least attractive and larger sizes $(14,16)$ were rated less attractive than smaller ones (6, 8, 10, 12; except for size 6 and 14). These findings were in agreement with previous observation that female bodies with a low but healthy BMI often attract the highest attractiveness ranking (Mo et al., 2013; Tovée et al., 1998). The fact that size 6 was rated similarly to size 14 suggested that thinner is not necessarily more attractive. Indeed, although a high BMI can be indicative of health problems, a very low BMI can also cause complications such as infertility (RichEdwards, 2002), thus reducing attractiveness and mate competition.

The effect of clothing on body attractiveness judgement was modulated by dress size (Fig. 2). The female body in smaller dress sizes $(6,8,10)$ were rated equally attractive regardless of clothing. Larger bodies $(12,14,16,18)$, however, were rated as more attractive if they were in loose clothing. A similar trend was also found for body size judgement (Fig. 3). Specifically, larger sizes $(10,12,14,16,18)$ in loose clothing were rated significantly smaller than in tight clothing. It therefore seems that for larger bodies, loose clothing can lead to an underestimation of body size and consequently increase body attractiveness ratings. This not only highlights the importance of unambiguous regional body cues for accurate body size estimation, but also extends to 
clothing retailers whom have the opportunity to utilise the fact loose fitting clothing is flattering for the larger individual, which could in turn be used to improve body satisfaction.

\subsection{Dress size and clothing type modulate body-viewing gaze allocation?}

When judging body attractiveness and body size, our participants viewed the waist-hip region significantly longer than other body areas (Fig. 4). This is consistent with previous literature that the waist-hip is an important determinant of attractiveness (Singh, 1993) and women attend to the mid and lower torso when assessing attractiveness (Cornelissen et al., 2009). However, Cornelissen et al. (2009) also found women gazed frequently at the chest, another region important for attractiveness (Singh \& Young, 1995), whereas we found no differences between viewing time at the upperbody and leg regions. This discrepancy might be caused by the clothing. The body's chest area was modestly covered in our study (even in the tight clothing condition), but was nude in Cornelissen et al. (2009) which might draw more attention to this area.

The similar amount of viewing time directed at the upper-body and legs suggested these regions may share equal importance in judging body attractiveness and body size, probably through providing visual cues about sexual maturity and body fat that are two important attractiveness factors (Singh, 1993; Smith, Cornelissen, \& Tovée, 2007). Interestingly, the viewing time allocated to the arms was significantly less than all other body regions, indicating its lack of relevance in judging female body attractiveness and size. As women tend to deposit a larger amount of fat onto the lower body parts (e.g., buttocks and thighs; Henss, 2000), these regions may contain more diagnostic cues for body attractiveness and body size assessment than other body regions such as the arms. It seems that in the context of mate selection theory (Buss, 2003), women predominantly attend to the body regions men looking for in a mate, 
probably to assess both the competition and their relative mate value.

As mentioned earlier, previous research has overlooked the influence of clothing on viewing behaviour, often using minimally clothed figures. Our manipulation of clothing type revealed some interesting findings. Compared to loose clothing, tight clothing significantly increased viewing time at the waist-hip region but decreased viewing at the legs (Fig. 4). It appears when the waist-hip (i.e. torso and hip area) was somewhat concealed by the loose clothing, causing ambiguity for its true size, the gaze was diverted away from this region to the legs which could still provide clear size and shape information and consequently indicate body mass. Clearly, the body regions are only attended to the extent they provide accessible information for the relevant body perception task (Bleske-Rechek, Kolb, Stern, Quigley, \& Nelson, 2014).

Whereas previous studies have analysed the gaze patterns for bodies representing the extremities of overweight and thin, this study used a continuum of body sizes. In contrast to clothing type, body size had limited impact on our participants' viewing behaviour. The larger dress size (UK 16 and 18) in loose clothing only slightly reduced the proportion of viewing time directed at the leg region. Perhaps the ambiguity of bodily cues caused by loose clothing is more evident for large dress sizes, forcing our participants to distribute gaze at multiple regions to rate body attractiveness and size.

\subsection{Individual differences in body-viewing gaze allocation?}

To explore the possible individual differences in body assessment related viewing behaviour in a non-clinical population, we correlated participants' own body composition and satisfaction measurements with their gaze distribution in viewing of preferred and least preferred bodies. The evident negative correlation between overall body satisfaction scores and PACS scores in our participants suggested those with 
lower body satisfaction undertake more body comparisons, and this internal-driven comparison process might be manifested in their body-viewing gaze allocation.

To our knowledge, this is the second study to specifically investigate the effect of body region satisfaction on body region viewing. Whereas Lykins et al. (2014) found self-satisfaction of a specific body region (e.g., the torso) would predict gaze allocated at that region, we found body region satisfaction was correlated with the viewing of alternative regions. Specifically, waist-hip satisfaction was positively correlated with the viewing time at the upper-body of both the preferred and least preferred body sizes. Additionally for preferred (thus idealised) body size, arm and leg satisfactions were positively correlated with viewing of the waist-hip and arm regions, respectively (Fig. 7). In relation to social comparison theory, it seems that in our sample of healthy young women, self-satisfaction with a body region means the need for comparing that region is reduced and thus gaze is allocated at the neighbouring body areas that are also informative for body attractiveness and size assessment, especially when viewing the preferred body image. The discrepancy between this study and previous research might be caused by participant groups. The reported attention or viewing biases towards the concerned body region were from participants scoring at the extreme ends of body dissatisfaction measures (Cho \& Lee, 2013) and having the greatest concern of a specific body area (Lykins et al., 2014). Our participants, however, were generally happy with their body compositions with indistinguishable satisfaction ratings across different body regions. Furthermore, considering that an observer's body-viewing gaze allocation can be modified by different task demands (Yarbus, 1967), the variance in task instruction between different studies (e.g., free-viewing task in Lykins et al. (2014) vs body attractiveness and body size judgement task in this study) might also lead to the inconsistent findings.

The effect of objective body composition measurements, such as chest size, on 
body-viewing gaze distribution has not previously been explored. Interestingly, in this study we noticed that participant's cup size was positively correlated with the viewing time at the upper-body of the preferred body image or in tight clothing (Fig. 5 and 6), despite no correlation being found between their self-reported satisfaction of own chest area and their viewing of others' chest. Therefore it seems having a smaller chest size may result in an unconscious avoidance of viewing other women's chest area, possibly to preserve self-esteem. This avoidance behaviour is similar to that found by Lykins et al. (2014) for torso satisfaction measurement, and it may allow the gaze to be distributed to less concerned body areas for downward comparison. Alternatively, this correlation might be also caused by women with a large cup size showing increased tendency to compare this body region with the others, and hence taking more interest in other women's chest area. Future research could address these two possibilities explicitly.

Regarding to other objective body composition measurements, BMI and own dress size were positively correlated with the viewing time allocated at the legs of the preferred body and the legs in tight clothing, respectively (Fig. 5 and 6). Since body fat is often deposited to women's lower body, the leg area would be affected by an increased BMI. Like having a small chest, a large BMI is generally seen as less attractive (Roef et al., 2008). However whereas smaller chest size tended to lead to avoidance viewing behaviour, larger BMI appeared to result in a gaze focus to areas of fat deposits suggesting an upward comparison. One explanation for this differing gaze 
behaviour may be that chest size is less changeable than BMI, and therefore women have learnt to avoid comparing themselves. BMI however is a more changeable construct, and therefore upward comparisons occur in an attempt for healthy selfimprovement.

Interestingly, in this study neither objective body composition measurements nor subjective body satisfaction scores correlated with the ratio between viewing time at the preferred and least preferred body images, whereas previous research has observed those with low body satisfaction attended longer to thin ideals than overweight bodies (Blechert et al., 2009; Cho \& Lee, 2013). This discrepancy may be caused by different image presentation methods. In the studies by Blechert et al. (2009) and Cho and Lee (2013) multiple bodies were presented simultaneously to compete for attention. In our study however, a single body was presented at a given trial. It may be that attention is only increased for idealised bodies when less attractive bodies are competing for attention. Additionally, participant group might also contribute to all the noticed differences between our and previous studies. Our participants were generally confident young female undergraduates who often had healthy below-average BMIs (Moody, 2012) and were wholly satisfied with their body (indicated by above-average body satisfaction scores). It would be interesting to run the same test on women across different age groups, in different professions, and with more varied BMI and body satisfaction scores to examine to what extent the current findings can be generalised to the wider non-clinical female population. It should be also noted that the body images used in this study were computer-generated avatars rather than real women, and the participants were required to rate both body attractiveness and body size in a given trial. Considering that an observer's scene-viewing gaze allocation can be modified by the perceived image quality (Röhrbein et al., 2015) and different task demands (Yarbus, 
1967), it would be interesting to repeat this study with photos of real women and with separate task instructions of judging body attractiveness and judging body size.

\subsection{Conclusion}

This study has demonstrated the clear impact of clothing and dress size on women's gaze behaviour in assessing female body attractiveness and body size, indicating the important role of clothing in mediating women's body perception. In contrast to tight clothing, loose clothing tended to divert gaze away from the concealed waist-hip area to the leg region in order to gain more accurate and available bodily cues, and subsequently led to decreased body size but increased body attractiveness ratings for large sized female bodies.

Own body satisfaction and body composition measurements also affected women's body-viewing gaze allocation, possibly through different cognitive processes. Subjective satisfaction of a specific body region was associated with the increased gaze towards neighbouring body areas, suggesting that satisfaction reduced the need for comparison with that body region. Objective measurements revealed that smaller chest size led to decreased viewing time at the chest area, but larger BMI and dress size led to increased viewing time at the leg region, implying healthy women may avoid comparing themselves on less changeable body regions in order to preserve self-esteem.

\section{References}

Buss, D. M. (2003). The evolution of desire: strategies of human mating (rev. ed.). New York, NY: Basic Books.

Blechert, J., Nickert, T., Caffier, D., \& Tuschen-Caffier, B. (2009). Social comparison and its relation to body dissatisfaction in bulimia nervosa: evidence from eye movements. Psychosomatic Medicine, 71, 907-912. 
Bleske-Rechek, A., Kolb, C. M., Stern, A. S., Quigley, K., \& Nelson, L. A. (2014). Face and body: independent predictors of women's attractiveness. Archives of Sexual Behavior, 43, 1355-1365.

Cho, A., \& Lee, J. H. (2013). Body dissatisfaction levels and gender differences in attentional biases toward idealized bodies. Body Image, 10, 95-102.

Cornelissen, P. L., Hancock, P. J., Kiviniemi, V., George, H. R., \& Tovée, M. J. (2009). Patterns of eye movements when male and female observers judge female attractiveness, body fat and waist-to-hip ratio. Evolution and Human Behavior, 30, 417-428.

Crossley, K. L., Cornelissen, P. L., \& Tovée, M. J. (2012). What is an attractive body? Using an interactive 3D program to create the ideal body for you and your partner. PloS One, 7(11): e50601.

Festinger, L. (1954). A theory of social comparison processes. Human Relations, 7, 117-140.

Fiske, S. T. (2011). Envy up, scorn down: How status divides us. New York: Russell Sage.

Glauert, R., Rhodes, G., Fink, B., \& Grammer, K. (2010). Body dissatisfaction and attentional bias to thin bodies. International Journal of Eating Disorders, 43, 4249.

Guo, K., Mahmoodi, S., Robertson, R. G., \& Young, M. P. (2006). Longer fixation duration while viewing face images. Experimental Brain Research, 171, 91-98.

Hahn, A. C., \& Perrett, D. I. (2014). Neural and behavioral responses to attractiveness in adult and infant faces. Neuroscience and Biobehavioral Reviews, 46, 591-603.

Hall, C., Hogue, T., \& Guo, K. (2011). Differential gaze behavior towards sexually preferred and non-preferred human figures. Journal of Sex Research, 48, 461-469.

Hall, C. L., Hogue, T., \& Guo, K. (2014). Sexual cognition guides viewing strategies to human figures. Journal of Sex Research, 51, 184-196.

Henss, R. (2000). Waist-to-hip ratio and female attractiveness. Evidence from photographic stimuli and methodological considerations. Personality and Individual Differences, 28, 501-513.

Horndasch, S., Kratz, O., Holczinger, A., Heinrich, H., Hönig, F., Nöth, E., \& Moll, G. H. (2012). "Looks do matter”- Visual attentional biases in adolescent girls with eating disorders viewing body images. Psychiatry Research, 198, 321-323. 
Hughes, S. M., Harrison, M. A., Gallup Jr., G .G. (2004). Sex differences in mating strategies: mate guarding, infidelity and multiple concurrent sex partners. Sexualities, Evolution and Gender, 6, 3-13.

Janelle, C. M., Hausenblas, H. A., Ellis, R., Coombes, S. A., \& Duley, A. R. (2009). The time course of attentional allocation while women high and low in body dissatisfaction view self and model physiques. Psychology and Health, 24, 351366.

Jansen, A., Nederkoorn, C., \& Mulkens, S. (2005). Selective visual attention for ugly and beautiful body parts in eating disorders. Behaviour Research and Therapy, 43, 183-196.

Jasieńska, G., Ziomkiewicz, A., Ellison, P. T., Lipson, S. F., \& Thune, I. (2004). Large breasts and narrow waists indicate high reproductive potential in women. Proceedings of the Royal Society: Biological Sciences, 271, 1213-1217.

Jiang, M. Y., \& Vartanian, L. R. (2012). Attention and memory biases toward bodyrelated images among restrained eaters. Body Image, 9, 503-509.

Lykins, A. D., Meana, M., \& Strauss, G. P. (2008). Sex differences in visual attention to erotic and non-erotic stimuli. Archives of Sexual Behavior, 37, 219-228.

Lykins, A. D., Ferris, T., \& Graham, C. A. (2014). Body region dissatisfaction predicts attention to body regions on other women. Body Image, 11, 404-408.

Mo, J. J., Cheung, K. W., Gledhill, L. J., Pollet, T. V., Boothroyd, L. G., \& Tovée, M. J. (2013). Perceptions of female body size and shape in China, Hong Kong, and the United Kingdom. Cross-Cultural Research, 48, 78-103.

Moody, A. (2012). Adult anthropometric measures, overweight and obesity. Health Survey for England. London: The Health and Social Care Information Centre.

Nummenmaa, L., Hietanen, J. K., Santtila, P., \& Hyönä, J. (2012). Gender and visibility of sexual cues influence eye movements while viewing faces and bodies. Archives of Sexual Behavior, 41, 1439-1451.

O’Connor, J. J. M., \& Feinberg, D. R. (2012). The influence of facial masculinity and voicepitch on jealousy and perceptions of intrasexual rivalry. Personality and Individual Differences, 52, 369-373.

Pawlowski, B., \& Dunbar, R. I. (1999). Impact of market value on human mate choice decisions. Proceedings of the Royal Society: Biological Sciences, 266, 281-285. 
Rich-Edwards, J. W., Spiegelman, D., Garland, M., Hertzmark, E., Hunter, D. J., Colditz, G. A., ... \& Manson, J. E. (2002). Physical activity, body mass index, and ovulatory disorder infertility. Epidemiology, 13, 184-190.

Roefs, A., Jansen, A., Moresi, S., Willems, P., van Grootel, S., \& van der Borgh, A. (2008). Looking good. BMI, attractiveness bias and visual attention. Appetite, 51, 552-555.

Röhrbein, F., Goddard, P., Schneider, M., James, G., \& Guo, K. (2015). How does image noise affect actual and predicted human gaze allocation in assessing image quality? Vision Research, 112, 11-25.

Rupp, H. A., \& Wallen, K. (2007). Sex differences in viewing sexual stimuli: an eyetracking study in men and women. Hormones and Behavior, 51, 524-533.

Singh, D. (1993). Adaptive significance of female physical attractiveness: role of waistto-hip ratio. Journal of Personality and Social Psychology, 65, 293-307.

Singh, D., \& Singh, D. (2011). Shape and significance of feminine beauty: an evolutionary perspective. Sex Roles, 64, 723-731.

Singh, D., \& Young, R. K. (1995). Body weight, waist-to-hip ratio, breasts, and hips: role in judgements of female attractiveness and desirability for relationships. Ethology and Sociobiology, 16, 483-507.

Smith, K. L., Cornelissen, P. L., \& Tovée, M. J. (2007). Color 3D bodies and judgements of human female attractiveness. Evolution and Human Behavior, 28, 48-54.

Stice, E., \& Shaw, H. E. (2002). Role of body dissatisfaction in the onset and maintenance of eating pathology: a synthesis of research findings. Journal of Psychosomatic Research, 53, 985-993.

Tatler, B. W. (2007). The central fixation bias in scene viewing: Selecting an optimal viewing position independently of motor biases and image feature distributions. Journal of Vision, 7(14), 1-17.

Thompson, J. K., Heinberg, L., \& Tantleff, S. (1991). The physical appearance comparison scale (pacs). The Behavior Therapist, 14, 174.

Tovée, M. J., Reinhardt, S., Emery, J. L., \& Cornelissen, P. L. (1998). Optimum bodymass index and maximum sexual attractiveness. The Lancet, 352, 548.

Weeden, J., \& Sabini, J. (2005). Physical attractiveness and health in western societies: a review. Psychological Bulletin, 131, 635-653.

Yarbus, A. (1967). Eye movements and vision. Plenum, New York. 


\section{Figure Legends}

Figure 1. Examples of female body images in UK dress size 6 (left) and 18 (right).

Figure 2. Attractiveness ratings for each dress size and clothing type. Error bars represent standard error of the mean.

Figure 3. Differences in perceived body size and actual dress size for each clothing type on each dress size. Error bars represent standard error of the mean.

Figure 4. Average proportion of viewing time directed at individual body regions in each dress size with loose clothing (left) and tight clothing (right). Error bars represent standard error of the mean.

Figure 5. Correlation between participants' chest size and viewing at upper-body region (left), and between dress size and viewing at leg region (right), for tight clothing only.

Figure 6. Correlation between participants' chest size and viewing at upper-body region (left), and between BMI and viewing at leg region (right) of the preferred body size.

Figure 7. Correlations between subjective body region satisfaction score and proportion of viewing time at body regions in the preferred body size (arm satisfaction and waisthip viewing, leg satisfaction and arm viewing, waist-hip satisfaction and upper-body viewing) and in the least preferred body size (waist-hip satisfaction and upper-body viewing). 